\title{
Reliability Study of RTV 566 for Its Application as a "Spring"
}

\author{
A. K. Dokania and P. Kruit
}

\begin{abstract}
This paper reports the preliminary feasibility study of room temperature vulcanization (RTV) 566 for its application as a "spring" for in situ height adjustment in our proposed design for an array of Schottky emitters. The reliability of the RTV 566 is tested in terms of its viscoelastic properties for thicknesses of 95, 142, and $195 \mu \mathrm{m}$. The shear modulus varied a little with the thickness. Although the RTV 566 shows a very little hysteresis, it shows a clear sign of viscoelastic behavior, rendering it unsuitable for the application. The viscoelastic behavior of the RTV 566 is modeled with the Findley model.
\end{abstract}

Index Terms-Reliability estimation, silicone rubber, strain measurement, stress measurement, time-domain analysis.

\section{INTRODUCTION}

$\mathbf{T}$ HE $\mathrm{Zr} / \mathrm{O} / \mathrm{W}(100)$ Schottky electron emitter is known for its high brightness and good emission stability [1] and is widely used in electron microscopes and electron-beam lithography systems. The Schottky emitter consists of a monocrystal tungsten (100) tip, which is spot welded on a heating filament of tungsten for heating up to $1800 \mathrm{~K}$. We are developing a miniaturized version of the Schottky emitter of $1 \mathrm{~mm}$ in diameter for a parallel electron lithography system. The alignment of the emitter tip with respect to various electrodes in an electron column is very critical. For thermomechanical reasons, the position of the tip in $x-y-z$-direction changes with time, which also alters the electron emission and the electron optics. For an array of hundreds of emitters, the differences in the electron optical properties between the individual emitter in the array must be reduced to a minimum. In a standard Schottky emitter, the tip displaces by $50 \mu \mathrm{m}$ in $z$-direction during its lifetime. To compensate this displacement, we propose an in situ position alignment of the tip using silicone-rubber room temperature vulcanization (RTV) 566.

RTV 566 has good thermal stability over a range of $-115{ }^{\circ} \mathrm{C}-260{ }^{\circ} \mathrm{C}$, low outgassing, and good adhesion with different set of materials [2]. RTV 566 is widely used in various mechanical and electronic engineering applications such as automotive heating hoses, chip bonding, solar cells, space application, and spark plug caps. The schematic of the proposed design to control the $z$-movement is shown in Fig. 1. In the

Manuscript received August 14, 2007; revised October 28, 2007.

A. K. Dokania is with the Faculty of Applied Sciences, Delft University of Technology, 2628 CJ Delft, The Netherlands (e-mail: a.k.dokania@tudelft.nl).

P. Kruit is with the Faculty of Applied Sciences, Delft University of Technology, 2628 CJ Delft, The Netherlands and also with the MAPPER Lithography B.V., 2628 CJ Delft, The Netherlands.

Color versions of one or more of the figures in this paper are available online at http://ieeexplore.ieee.org.

Digital Object Identifier 10.1109/TDMR.2008.915627

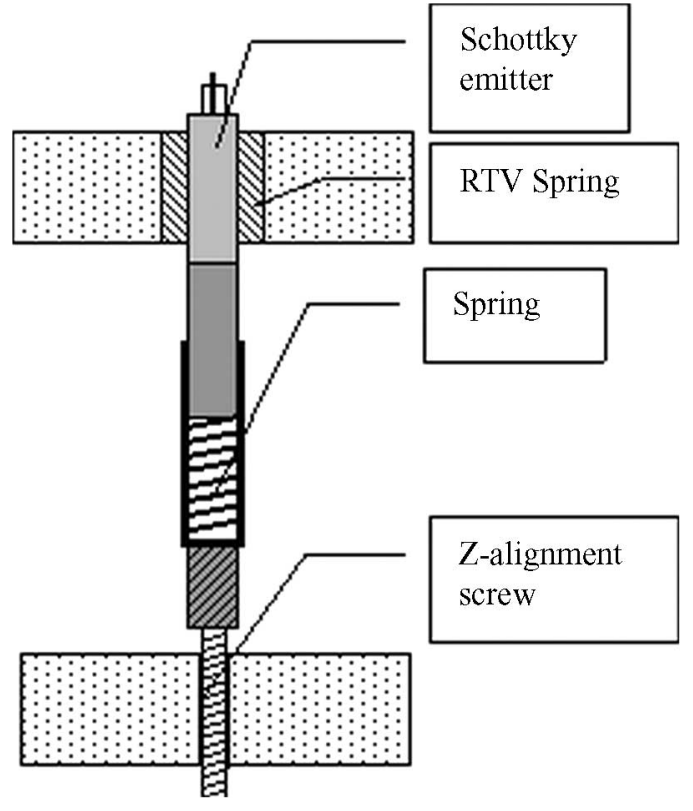

Fig. 1. Schematic of the design for in situ $z$-actuation.

proposed design, the Schottky emitter would be fixed to a base plate using the RTV 566 in a vacuum, and the emitter unit is attached to the spring-screw system which would impart force for the $z$-displacement.

However, silicone rubbers, like other elastomers, may have a viscoelastic behavior, resulting in hysteresis and creep. For the reliability of the RTV 566 in the bond, its creep and aging behaviors have to be considered, and a predictable stress-strain behavior of the RTV 566 is desired. There are some literatures that discuss the aging phenomenon of RTV silicone rubber [3]-[5]. However, in these papers, the aging phenomenon had been discussed in "extreme environment," i.e., corrosive, humid, or electric field. In [6], O'Sullivan et al. discuss the creep behavior of RTV 9161.

Moreover, different RTV silicone-rubber series, which are specified by the numbers, use different resins in it; therefore, each series of the RTV silicone rubber has a unique set of properties. Since no detailed viscoelastic study has been done for RTV 566, a preliminary viscoelastic study of the RTV 566 is carried out by measuring the shear-stress-shearstrain curve, the time-dependent force-displacement curve, and the strain at constant stress to assess the viscoelastic behavior. In this paper, we report the measured shear modulus for different thicknesses of the RTV 566 and its viscoelastic behavior under shear stress at room temperature of $21^{\circ} \mathrm{C}$. The RTV 566 shows a negligible hysteresis. However, 
a viscoelastic property of RTV 566 is observed, which is modeled with the Findley model at room temperature. An exact predictable model for the creep phenomenon would need an extensive experiment at different temperatures and stress levels.

\section{MODEL DESCRIPTION}

The schematic shown in Fig. 1 is based on the model described next. The tip is placed in a guide using the RTV 566. Behind the guide, a spring is placed to actuate the tip. The $z$-displacement by the screw is demagnified by the ratio of stiffness of the spring and the RTV $566\left(C_{\mathrm{s}} / C_{\mathrm{RTV}}\right)$. Stiffness calculations are done for the RTV 566 and the spring. Finally, the resolution that can be achieved is calculated.

The resolution of a screw is determined by the ease of rotation of the screw. By assuming a rotation of $10^{\circ}$ and the slew of $0.2 \mathrm{~mm}$, a resolution that can be achieved by the screw $\left(R_{\text {screw }}\right)$ is $5.556 \mu \mathrm{m}(0.2 \mathrm{~mm} \times 10 / 360)$.

The resolution of the tip depends on the resolution of the screw and the ratio of stiffness of the spring and the RTV 566, which is given by

$$
R_{\text {tip }}=R_{\text {screw }} \cdot \frac{C_{\mathrm{s}}}{C_{\mathrm{RTV}}} .
$$

The stiffness for the RTV 566 and the spring is given by

$$
\begin{aligned}
C_{\mathrm{RTV}} & =\frac{2 \cdot \pi \cdot l \cdot G_{\mathrm{RTV}}}{\ln \left(\frac{d_{\mathrm{o}}}{d_{\mathrm{i}}}\right)} \\
C_{\mathrm{s}} & =\frac{G_{\mathrm{s}} \cdot d^{4}}{8 \cdot D^{3} \cdot n}
\end{aligned}
$$

where $G_{\mathrm{RTV}}$ and $G_{\mathrm{S}}$ are the shear moduli of the RTV 566 and the spring, respectively, $d_{\mathrm{i}}$ is the diameter of the emitter, $d_{\mathrm{o}}$ is the diameter of the hole in the block, $d$ is the wire diameter of the spring, $D$ is the diameter of the helix for spring, and $n$ is the number of coil in the spring. The small thickness of applied RTV 566 would result in high stiffness which can translate the screw movement to a very small movement of the tip. For a typical aluminum spring of stiffness $0.7 \mathrm{~N} / \mathrm{mm}$ and RTV 566 thickness of $95 \mu \mathrm{m}$, a resolution of $45 \mathrm{~nm}$ can be achieved. Different thicknesses of the RTV 566 would give different resolutions, and depending upon the frequency of adjustment (daily or weekly) during the lifetime, appropriate resolution can be set by a proper choice of the RTV 566 thickness and choice of the spring.

The lifetime of such a system is expected to be a year, which is the same as the lifetime of the Schottky emitter. In the following sections, reliability study of the RTV 566 is discussed for a maximum stroke of $40 \mu \mathrm{m}$, which corresponds to the approximate change in position of the Schottky emitter during its lifetime, for different thicknesses of the RTV 566.

\section{EXPERIMENTAL METHODS}

Stainless-steel (SS) pins of $0.9 \mathrm{~mm}$ in diameter were used as a dummy Schottky emitter, which were fixed with the RTV 566 to the holes of different diameters in the 5-mm-thick SS block. This configuration closely represents our proposed
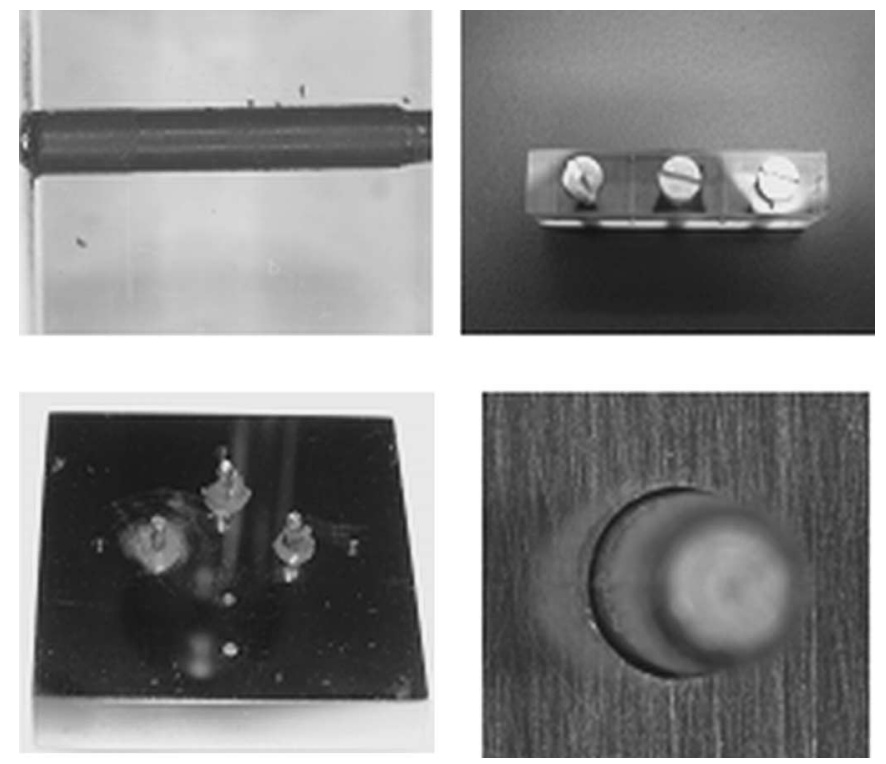

Fig. 2. (Top row) Smearing of the RTV 566 on SS pin and setting in the mold. (Bottom row) Pins in the SS block and final cured pin in the block.

real experimental setup, as shown in Fig. 1. The pins were fixed to the block by a Nederlandse Organisatie voor toegepast natuurwetenschappelijk (TNO) proprietary "two-phase" curing process. First, the pins and the holes in the block were cleaned, and 2-3 $\mu \mathrm{m}$ of supplier-prescribed SS4155 primer was coated and cured. Then, the thin layer of RTV 566 was smeared around the pin in the mould and cured, as shown in Fig. 2 (top row). The mould was used to create a homogeneous thin film of the RTV 566 around the pin with different thicknesses and to avoid air encapsulation. Three different thicknesses, i.e., 95, 142, and $190 \mu \mathrm{m}$, of the RTV 566 layer were coated over the pin. In the next step, the pin with a cured RTV 566 layer and the hole in the base block were wetted with the RTV 566. Thereafter, the pins were inserted into the hole, centered carefully, and cured, as shown in Fig. 2 (bottom row). The aforementioned process gave rise to the fixing of the pins with different thicknesses of the RTV 566 to the block.

An unsuccessful attempt to fix the pins to the block with the RTV 566 by a vacuum-suction method was also made. However, this method gave rise to the formation of air bubbles.

The load on the pin is applied by a weighing gauge to give a sufficient displacement to the pin. This load corresponds to the load that would be acting on the tip by the screw action, as shown in Fig. 1. The schematic of the loading and displacement of the RTV 566 layer in the block is shown in Fig. 3(a), where $F$ denotes the force applied, $l$ is the thickness of the block, $s$ is the displacement of the RTV 566 layer, $d_{\mathrm{i}}$ is the diameter of the SS pin, and $d_{\mathrm{o}}$ is the diameter of the hole in the block. In this setup, the sensor needles were placed on the top and the bottom of the pin, as shown in Fig. 3(b). These sensor needles were attached to a displacement meter with sensitivity in opposite directions, which compensates the small vibrations and displacement of the pin-block system, if any. Then, a small push (few grams of weight) was applied on the top needle by the weighing gauge, and the displacement of the tip was then indicated in the displacement meter. For a given weight, 


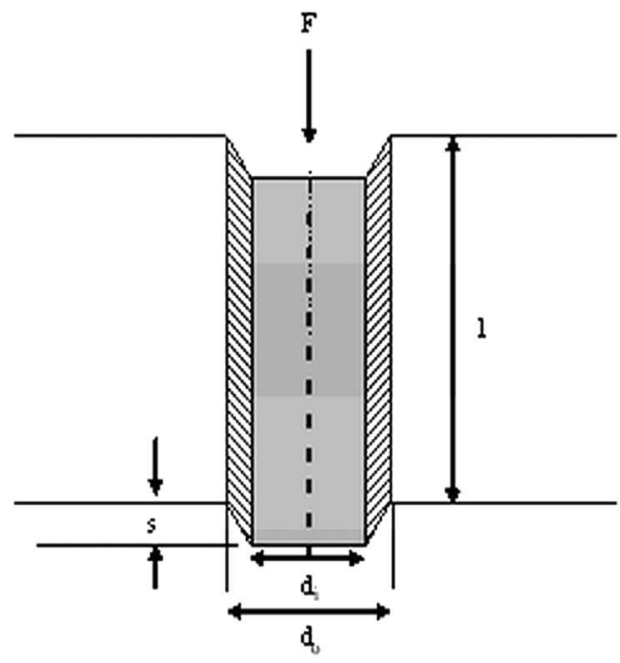

(a)

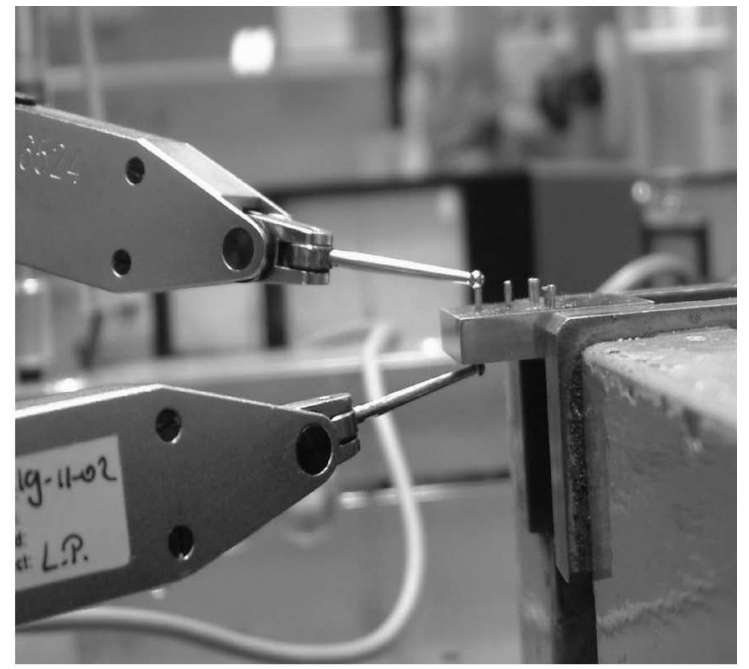

(b)

Fig. 3. (a) Schematic diagram of the pin in the hole with force acting on it. (b) Displacement sensors on the top and the bottom of the pin.

the displacement was measured for different thicknesses of the RTV 566. The applied load was gradually increased until the displacement of $40 \mu \mathrm{m}$ is obtained. The test was carried out in air and in a temperature-controlled room of $21^{\circ} \mathrm{C}$. The actual setup would be in a vacuum $\left(1-10^{-9} \mathrm{mbar}\right)$, and the cooling mechanism would keep the temperature of the base plate at $300 \mathrm{~K}$.

\section{RESUlTS AND Discussion}

We measured the shear modulus of elasticity for different thicknesses of the RTV 566 by determining the force needed for certain displacements. A hysteresis cycle was followed to check the possibility of any plastic deformation. The force and displacement was converted into stress and shear strain, as shown in Fig. 4. The values of shear modulus from our samples were obtained by fitting the straight line to the curves in Fig. 4, which shows the values of $0.5\left(500000 \mathrm{~N} / \mathrm{m}^{2}\right), 0.64$, and $0.635 \mathrm{MPa}$ for 95,142 , and $190 \mu \mathrm{m}$ of the RTV 566 thickness, respectively. The different values of shear modulus can be attributed to the different thicknesses and, thus, the different form factors [6] of the RTV 566. The form factor for this configuration can be calculated by the expression: $\left(d_{\mathrm{o}}-d_{\mathrm{i}}\right) / 4 l$, and the form factors are $0.0095,0.0124$, and 0.019 for 95 , 142 , and $190 \mu \mathrm{m}$ of the RTV 566, respectively. In general, specimens with large form factor will have a higher effective modulus than the associated bulk or shear modulus [6]; this may explain the higher shear modulus of $142-$ and $190-\mu \mathrm{m}$ samples. The variation of shear modulus could also be due to the variation in surface roughness, causing a variation in contact between the pin and the plate. The obtained value of the shear modulus is comparable to the elastic modulus of $0.552 \mathrm{MPa}$ reported in [7]. Due to the very low form factor of the RTV 566, the shear stress could be approximated to the tensile stress, and therefore, the shear and elastic moduli seem to be comparable.

In the reported range of the strain value, there is a negligible hysteresis observed in all the samples. No hysteresis was observed until $10 \%$ of shear-strain level for $95-\mu$ m-thick RTV 566. For 142- and 195- $\mu$ m-thick sample, hysteresis values between $6 \%$ and $7 \%$ is observed at the shear-strain value greater than $5 \%$. This could be due to the lower form factor of the 95- $\mu \mathrm{m}$ sample compared with the other samples. Low hysteresis for low form-factor samples was also observed in RTV 9161 [6]. No considerable hysteresis is observed for small deformation.

Fig. 5 shows the isochronous force-displacement curve where measurements were done on two values of elapsed times. The force (or stress) required to have the same displacement (or strain) is reduced with time. Although the sample was stored in an airtight container, it seems that the degradation of RTV 566 due to possible moisture absorption could not be avoided. A similar observation was reported for polydimethylsiloxane in [6]. However, for application in vacuum, aging due to moisture and other environmental factors could be avoided and might give better results.

There are no literature data available for the viscoelastic behavior of RTV 566, and thus, an attempt is made to analyze the viscoelastic behavior of the RTV 566. To model the viscoelastic property of RTV 566, a load of $9 \mathrm{~g}$ is applied to the pin with the RTV 566 of $142-\mu \mathrm{m}$ thickness to give an initial displacement of $1 \mu \mathrm{m}$, and the change in this displacement is recorded with time at constant stress. The result is plotted in the graph shown in Fig. 6. The curve is similar to the curve of a typical viscoelastic material. It can be seen from the curve that the RTV 566 has a nonlinear viscoelastic shear-strain characteristic even at low stress. The empirical data were fitted with the Findley model which is a nonlinear viscoelastic power-law creep model valid for a wide range of polymers. Findley's model [8] expresses the total strain as the sum of time-independent elastic and plastic strain and time-dependent viscous strain

$$
\varepsilon(t)=\varepsilon_{\mathrm{o}}+\varepsilon_{\mathrm{c}} t^{m}
$$

where $\varepsilon$ is the total strain, $\varepsilon_{\mathrm{o}}$ is the stress-dependent time-independent initial elastic and plastic strain, $\varepsilon_{\mathrm{c}}$ is the stress-dependent coefficient of the time-dependent viscous strain, $t$ is the time, and $m$ is the time exponent. 


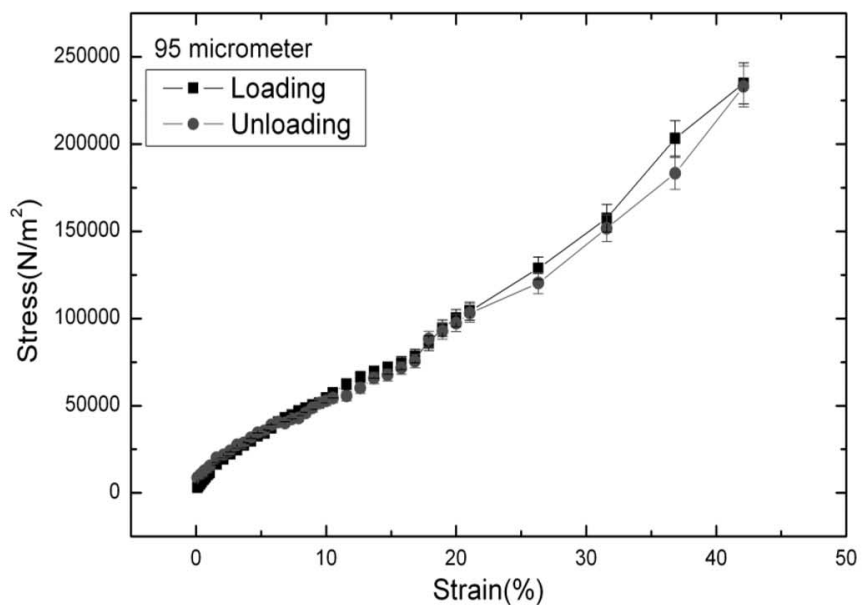

(a)

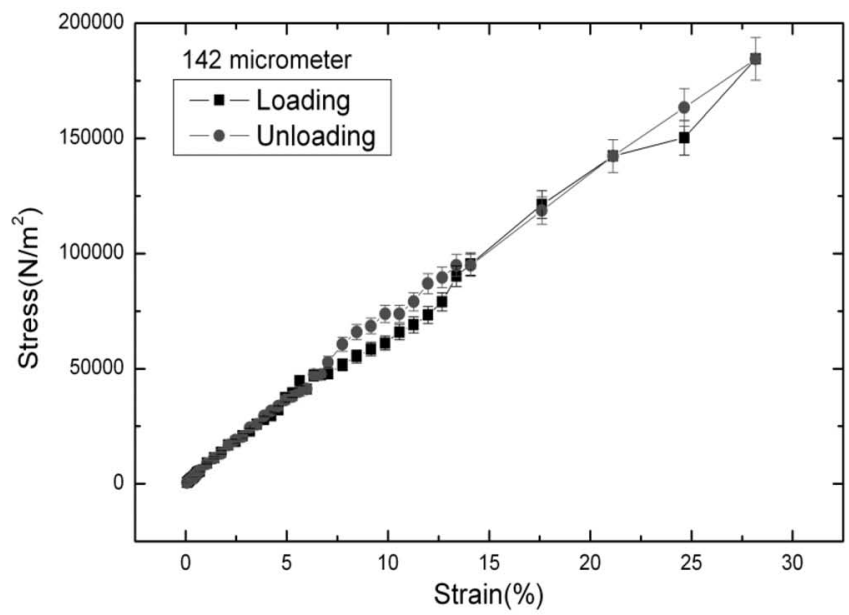

(b)

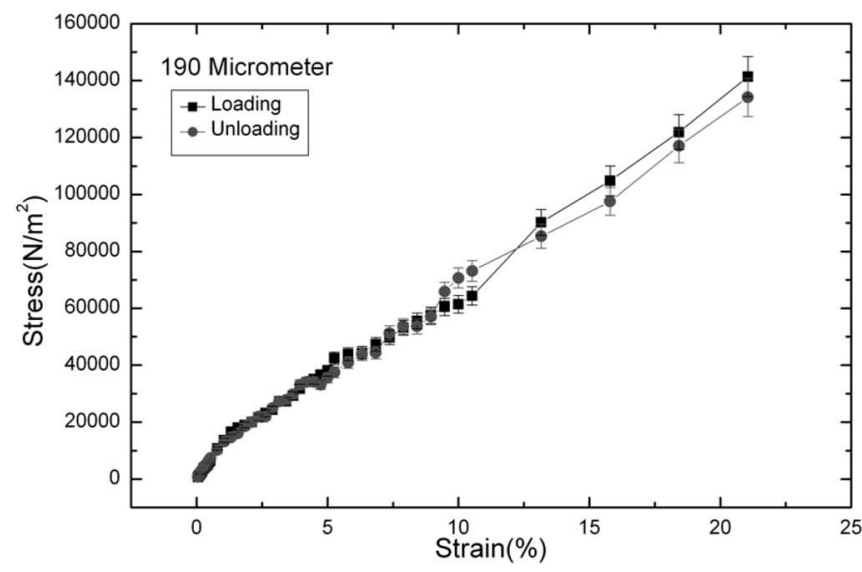

(c)

Fig. 4. Loading-unloading cycle of the RTV 566 for the thicknesses of (a) $95 \mu \mathrm{m}$, (b) $142 \mu \mathrm{m}$, and (c) $190 \mu \mathrm{m}$. Error bars: $5 \%$.

Based on the Findley model, curve fitting is done for the curve shown in Fig. 6, and the various coefficients obtained are as follows: $\varepsilon_{\mathrm{o}}$ is $0.00689, \varepsilon_{\mathrm{c}}$ is $4.87 \times 10^{-7}$, and $m$ is 0.6943 . The value of $m$ close to one denotes the strong time dependence of the total strain.

Although we did not have enough experimental data points to create a predictable creep model, these data give a qualitative insight into the creep phenomenon of the RTV 566. The

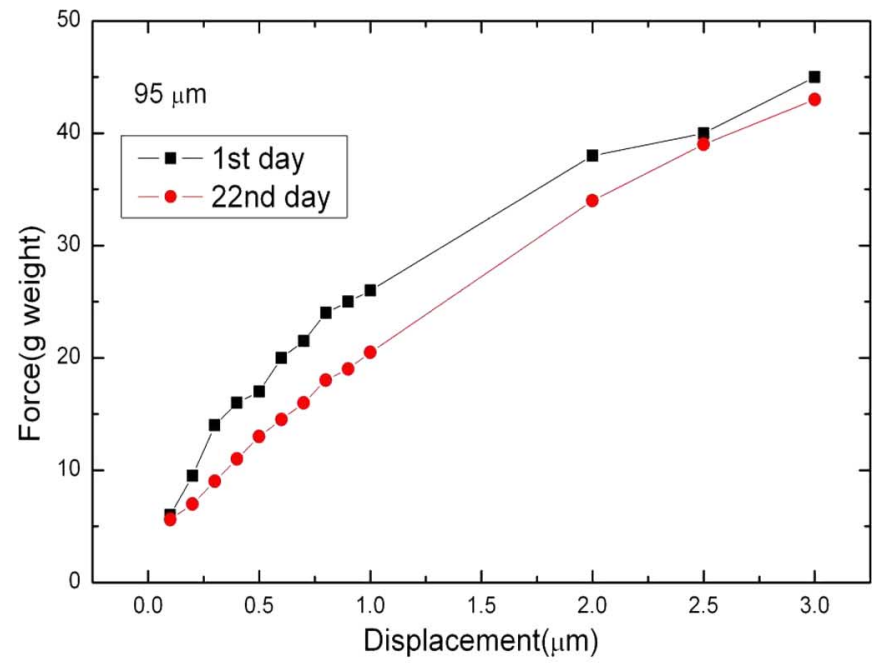

(a)

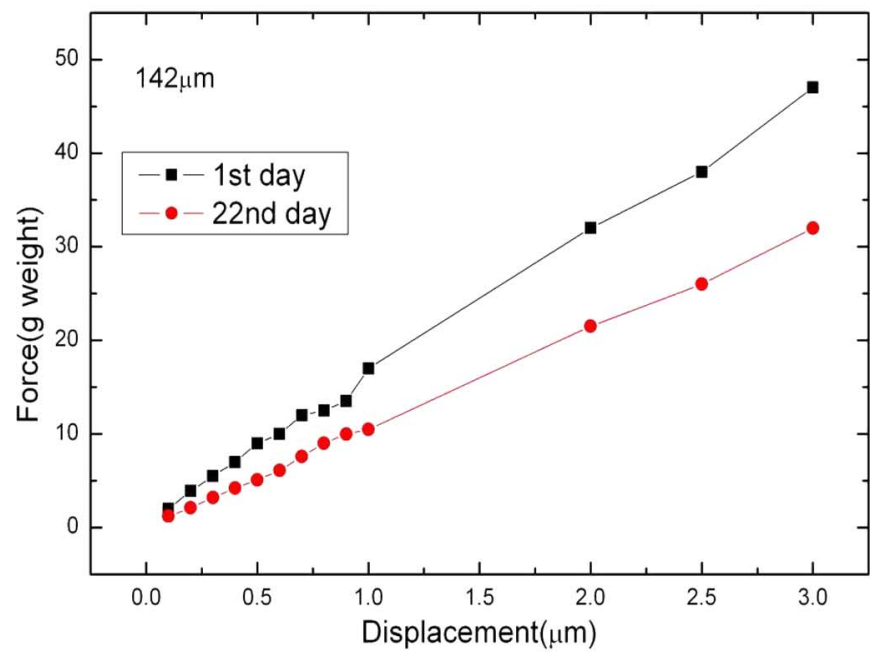

(b)

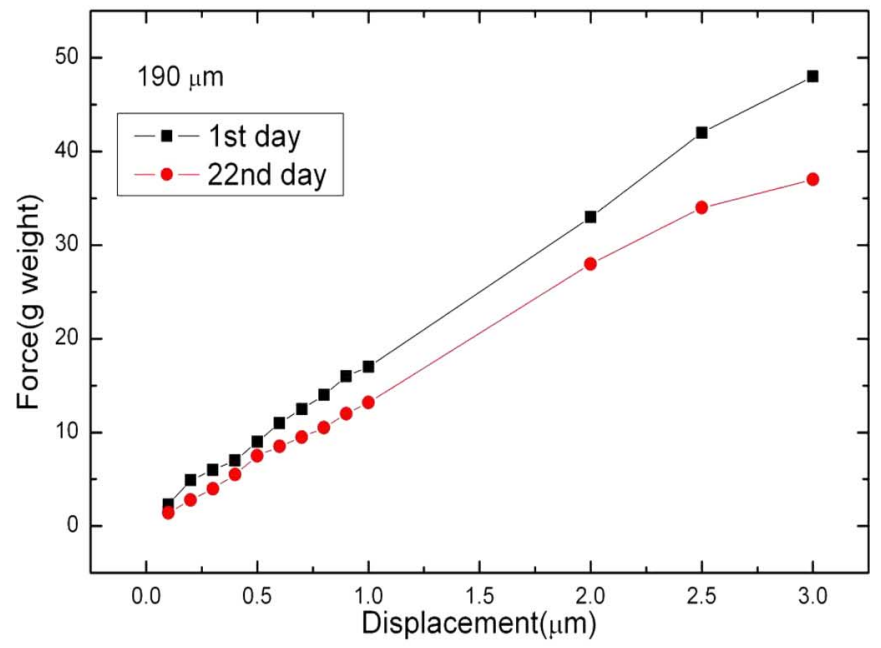

(c)

Fig. 5. Force-displacement curve of the RTV 566 performed on two values of elapsed times for (a) $95 \mu \mathrm{m}$, (b) $142 \mu \mathrm{m}$, and (c) $190 \mu \mathrm{m}$.

determination of time dependence of the force required for a certain displacement necessitates considerably more experimental data under various conditions of stress and temperature, which would avoid frequent calibrations. 




Fig. 6. Shear strain versus time for the load of 9-g weight for $142-\mu \mathrm{m}$-thick RTV 566.

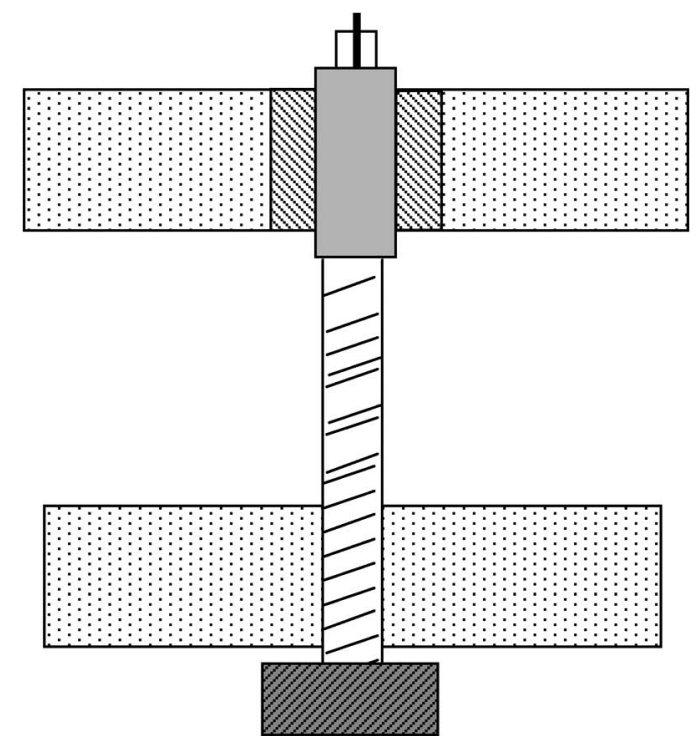

Fig. 7. Modification of the design shown in Fig. 1.

We assume that a material which can meet our requirement with no viscoelastic property would be difficult to find and that the use of such a material would require extensive study of its creep behavior to avoid calibration. Therefore, the design in which the RTV 566 only acts as a binder and not spring, as shown in Fig. 7, would be a feasible solution. In this design, the emitter would be placed over the fixed base of the screw, and the resolution of the tip then equals that of the screw. Another idea would be the use of linear variable differential transformer (LVDT) in place of screw. In both of these cases, the pitch of the adjustment depends only on the pitch of the screw or the LVDT attached.

\section{CONCLUSION}

A study has been done to check the feasibility of using an RTV 566 "spring" for in situ precision displacement in an experimental setup. The RTV 566 can be applied to an SS pin with a good thickness control. Loading and unloading can be done reliably with a negligible hysteresis. It is possible to have

a $z$-movement with RTV 566 in the elastic range. The timedependent deformation seems to follow the Findley model. However, its viscoelastic behavior can give rise to unpredictable behavior, making it unsuitable for the application in its current form. A different design is proposed to overcome this problem.

\section{ACKNOWLEDGMENT}

The authors would like to thank A. Vis, P. Giesen, and W. van Helden of the TNO for their technical support and collaboration.

\section{REFERENCES}

[1] L. W. Swanson and N. A. Martin, "Field electron cathode stability studies: Zirconium/tungsten thermal-field cathode," J. Appl. Phys., vol. 46, no. 5, pp. 2029-2050, May 1975.

[2] GE Silicones, RTV 566 Specification Sheets. [Online]. Available: http://www.gesilicones.com/siliconesweb/am1/en/documents/datasheets/ 1469.html

[3] S. H. Kim, E. A. Cherney, and R. Heckam, "The loss and recovery of hydrophobicity of RTV silicone rubber insulator coatings," IEEE Trans. Power Del., vol. 5, no. 3, pp. 1491-1500, Jul. 1990.

[4] D. Devendranath, N. S. Mohan Rao, Channakeshava, and A. D. Rajkumar, "Ageing studies on RTV coated insulator in salt-fog pollution," in Proc. IEEE Power Eng. Soc. Winter Meeting, 2000, vol. 4, pp. 2822-2829.

[5] H. Homma, C. L. Mirley, J. Ronzello, and S. A. Boggs, "Field and laboratory aging of RTV silicone insulator coatings," IEEE Trans. Power Del., vol. 15 , no. 4, pp. 1298-1303, Oct. 2000.

[6] S. O'Sullivan, R. Nagle, J. A. McEwen, and V. Casey, "Elastomer rubbers as deflection elements in pressure sensors: Investigation of properties using a custom designed programmable elastomer test rig," J. Phys. D, Appl. Phys., vol. 36, no. 15, pp. 1910-1916, Aug. 2003.

[7] S. Magill, M. MacLean, J. Schetz, R. Kapania, A. Sang, and W. Pulliam, "Study of direct-measuring skin-friction gauge with rubber sheet for damping," AIAA J., vol. 40, no. 1, pp. 50-57, Jan. 2002.

[8] W. N. Findley, "Mechanism and mechanics of creep in plastics," SPE J., vol. 16 , no. 10 , pp. 57-65, 1960.

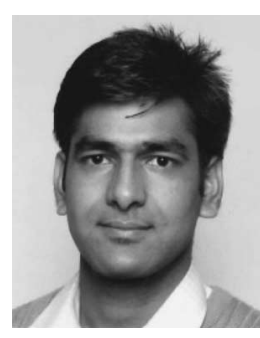

tion, and thin films.

Mr. Dokania is a nominated member of the American Association for the Advancement of Science.

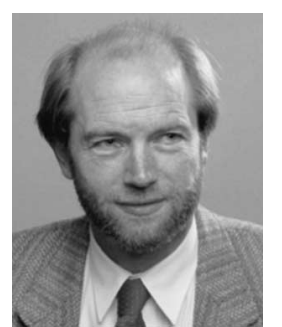

P. Kruit studied physics at the Delft University of Technology, Delft, The Netherlands, and received the Ph.D. degree from the University of Amsterdam, Amsterdam, The Netherlands.

He is a Full Professor of physics with the Faculty of Applied Sciences, Delft University of Technology. He has been the Chair of Charged Particle Optics since 1989. He is also with the MAPPER Lithography B.V., Delft, which is a company that he founded in 2000 . His current research interests include electron and ion optics, high-throughput e-beam lithography, sub-20-nm e-beam lithography, novel electron and ion sources, and microelectromechanical-system applications in electron optics.

Dr. Kruit has been, among other responsibilities, the Editor of Ultramicroscopy, the Chairman of the Fifth International Conference on Charged Particle Optics, the Program Chairman of MNE2004, the President of the Dutch Society for Microscopy, and the Department Head of Applied Physics. 